\title{
Études/Inuit/Studies
}

\section{Rectitude politique ou rectitude linguistique? Comment orthographier «Inuit» en français}

\section{Louis-Jacques Dorais}

Volume 28, numéro 1, 2004

Art et représentation

Art and representation

URI : https://id.erudit.org/iderudit/012644ar

DOI : https://doi.org/10.7202/012644ar

Aller au sommaire du numéro

Éditeur(s)

Association Inuksiutiit Katimajiit Inc.

ISSN

0701-1008 (imprimé)

1708-5268 (numérique)

Découvrir la revue

Citer cet article

Dorais, L.-J. (2004). Rectitude politique ou rectitude linguistique? Comment orthographier «Inuit» en français. Études/Inuit/Studies, 28(1), 155-159.

https://doi.org/10.7202/012644ar d'utilisation que vous pouvez consulter en ligne. 


\title{
Débats / Debates
}

\section{Rectitude politique ou rectitude linguistique? Comment orthographier «Inuit» en français}

\author{
Louis-Jacques Dorais
}

Tempête dans un verre d'eau? Découpage de cheveux en quatre? Depuis plusieurs années, un débat aussi feutré qu'apparemment sans importance oppose les tenants de deux écoles: ceux qui croient qu'en français, l'orthographe du mot «Inuit» devrait respecter le génie de la langue dont il est tiré, l'inuktitut, et ceux qui jugent qu'au contraire, les utilisateurs de ce terme devraient se plier aux règles de la grammaire française. Autrement dit, certains - que nous appellerons les inuitophiles - prônent l'invariabilité du vocable en question («des femmes inuit»), alors que d'autres - les francocentristes - considèrent qu'il devrait s'accorder en genre et en nombre, comme le font la plupart des mots de la langue française écrite («des femmes inuites»). Les inuitophiles se subdivisent eux-mêmes en «purs et durs,» qui emploient le mot «Inuk» comme singulier du nominal ou de l'adjectif appliqué à une personne («un(e) Inuk, des Inuit, un(e) artiste inuk, des artistes inuit»), et en «vacillants,» qui sont prêts à admettre l'usage d' «Inuit» au singulier («un(e) Inuit, un(e) artiste inuit»).

Derrière ces querelles byzantines se profile une opposition plus profonde entre tenants de la rectitude politique et partisans de la rectitude linguistique. Les premiers dont fait partie l'équipe de rédaction d'Études/Inuit/Studies - jugent que le respect des populations inuit doit nécessairement entraîner l'usage correct (selon les standards orthographiques qu'elles se sont elles-mêmes donnés) des mots tirés de leur langue, que ces mots aient été empruntés par le français, l'anglais ou tout autre idiome. Les seconds par contre croient en la primauté absolue des règles d'accord du français quand on parle et écrit cette langue bien sûr - quelle que soit l'origine des termes auxquels elles s'appliquent.

Pour mieux comprendre les tenants et aboutissants de ce débat, revenons un peu en arrière. C'est à partir de la fin des années 1960 et du début des années 1970, sous la pression des organismes politiques inuit alors en émergence, que le français et d'autres langues ont commencé à remplacer le mot «Esquimau» par le terme «Inuit.» Au début semble-t-il, la question orthographique ne se posait pas vraiment. La majorité des

Département d'anthropologie, Université Laval, Québec (Québec), Canada, G1K 7P4. louisjacques.dorais@ant.ulaval.ca

ETUDES/INUIT/STUDIES, 2004, 28(1): 155-159 
spécialistes et du public utilisaient le mot de façon invariable. Dans un article originellement publié en 1974 (Dorais 1974) et reproduit l'année suivante (Dorais 1975) dans une revue grand public, je tentais de formuler les règles d'usage qui semblaient être en train de s'imposer dans le monde francophone: invariabilité du mot «Inuit / inuit» en genre et en nombre, mais usage d' «Inuk» comme nom singulier et d'«inuk» comme adjectif singulier appliqué à une personne. Les exemples qui suivent (adaptés de Dorais 1975: 40) résument ces règles:

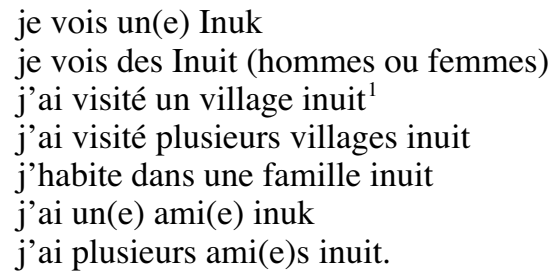

En 1979 (décision 85, datée du 14 décembre), l’Office de la langue française (OLF) du Québec, un organisme public ayant pour mission d'émettre des avis en matière d'usage et d'orthographe du français, entérinait la presque totalité de ces règles en décrétant que le nom des Inuit devait rester invariable en genre au singulier («Inuk») comme au pluriel («Inuit»), l'adjectif étant toujours pluriel («inuit») et invariable. Cette façon de faire respectait le génie de l'inuktitut, qui ne possède ni masculin ni féminin et où le pluriel est ordinairement exprimé par le suffixe -it (Dorais 1998) ${ }^{2}$. Devenue officielle au Québec, l'orthographe ainsi proposée fut rapidement adoptée par les services fédéraux canadiens, les médias d'information et même, dit-on, l'Académie française (Bernard Saladin d'Anglure, communication personnelle).

Or en 1993, un linguiste de l'OLF s'avisa que cette manière d'écrire était linguistiquement incorrecte et faisait injure à la langue française, qui met des $s$ au pluriel et des $e$ au féminin ${ }^{3}$. Sans consulter les Inuit (dont plusieurs, au Nunavik tout au moins, sont maintenant francophones) ni les spécialistes de leur langue et de leur culture, ce fonctionnaire décida qu'il faudrait désormais écrire: un Inuit, une Inuite, des Inuits ou Inuites, des villages inuits, des personnes d'origine inuite, des coutumes inuites, etc. L'orthographe proposée devint officielle quelques mois plus tard (OLF 1993) et depuis, elle s'est imposée dans les publications gouvernementales et les médias, tout au moins de façon partielle. On peut en effet noter que si la plupart des journalistes et autres rédacteurs n'hésitent pas à mettre un $s$ au mot «Inuit,» ils sont

L'usage du pluriel «inuit» comme qualificatif s'appliquant à une entité singulière autre qu'une personne se justifie par le fait que dans ce cas, l'adjectif signifie: «qui relève des Inuit.» Ainsi, l'expression «village inuit» ne doit pas être comprise comme signifiant «village qui est un Inuk,» mais «village relevant des Inuit.»

2 L'inuktitut possède un nombre duel (inuuk; «deux Inuit»), mais, d'une part, celui-ci est trop éloigné de nos habitudes grammaticales pour qu'on en tienne compte en français, d'autre part, il a maintenant disparu des dialectes groenlandais.

3 On peut s'interroger sur le pourquoi de cette soudaine prise de conscience. Rappelons qu'au Québec, le début des années 1990 fut marqué par le rejet de propositions fédérales (accords dits du Lac Meech et de Charlottetown) visant à donner à la province un statut particulier au sein de la confédération canadienne. Ce rejet entraîna une montée du nationalisme québécois francophone, qui allait aboutir à l'élection d'un gouvernement souverainiste en 1994 et à un référendum sur l'indépendance du Québec en 1995. Le changement de cap de l'OLF serait-il indirectement lié à ce contexte idéologique et politique? 
plus hésitants à lui adjoindre un $e$ final, ce qui dénote une certaine ambiguïté face aux nouvelles règles proposées. Le logiciel de correction de l'ordinateur que j'ai utilisé pour écrire ce texte accepte le mot «Inuits,» mais il souligne en rouge les formes «inuits,» «inuite,» «inuites» et, curieusement, «Inuk» et «inuk.»

La décision prise en 1993 par l'OLF souleva l'opposition de plusieurs Inuit et spécialistes de leur langue et de leur culture. Réunis à Iqaluit en juin 1994 à l'occasion du neuvième Congrès d'Études Inuit, il rejetèrent à l'unanimité la réforme orthographique proposée par l'Office, la considérant irrespectueuse et dérogatoire à l'égard du peuple inuit. Dans les années qui suivirent, un grand nombre d'Inuit francophones du Québec exprimèrent leur malaise face à des lettres ou autres textes en provenance du gouvernement qui, à leurs yeux, massacraient l'orthographe de leur ethnonyme (communication personnelle de Jacqueline Dorman, cadre supérieur au gouvernement du Québec).

Les inuitophiles, défenseurs d'une rectitude politique qui se veut soucieuse du respect des autres, refusèrent de se plier aux suggestions de l'OLF, arguant que, d'une part, la langue française ne propose pas de règles précises pour orthographier les noms de peuples étrangers - on écrit bien «les Kanak» et «les Atikamekw,»sans $s$ final et que, d'autre part, le respect de la langue et de la culture inuit passe bien au-dessus de considérations orthographiques un peu étroites. À l'heure actuelle, on observe que les spécialistes francophones des Inuit parlant eux-mêmes l'inuktitut ou le kalaallisut groenlandais considèrent toujours les mots «Inuk» et «Inuit» comme invariables en français. On peut en donner pour preuve les travaux de Robbe (1994), Csonka (1995), Dorais (1996), Therrien (1999), Saladin d'Anglure (Nappaaluk 2002) ou Huret (2003). Et il ne sont pas les seuls, bien d'autres auteurs (Mennecier 1995 et Laugrand 2002 par exemple) se rangeant eux aussi dans le camp des inuitophiles.

De leur côté, les francocentristes continuent à défendre leur propre logique de rectitude linguistique, tout en refusant de la pousser jusqu'en ses limites ultimes. En effet, la francisation du mot «Inuit» pose un problème phonétique. En français, la voyelle $u$ se prononce comme le $\ddot{u}$ allemand et le $t$ final est muet. Les francocentristes purs et durs devraient donc prononcer la seconde partie du mot «Inuit» de la même façon que le terme «nuit.»C'est ce que font d'ailleurs spontanément plusieurs journalistes de la radiotélévision et autres locuteurs du français, qui parleront des inüi ou des inüite. Il vont cependant encore rarement aussi loin que ce journaliste de la télévision française entendu il y a quelques mois, qui parlait des «Crisses et Inüitses du Nord québécois.» Il oubliait les Innüsses, les Naskapisses et les Atikamekwisses.

Tout ceci pour dire que les tenants de la rectitude linguistique ne sont pas complètement logiques avec eux-mêmes. Ils francisent l'usage grammatical du mot «Inuit»sans en franciser la phonétique. Interrogé à ce sujet, le linguiste de l'OLF responsable du dossier mentionnait à l'époque de vagues critères liés à l'usage orthographique pour expliquer le fait qu'on n'ait pas poussé le respect du français jusqu'au bout, en écrivant «Inouïte» au lieu d' «Inuit» (Bergeron 1994). Quelques années plus tôt, des chercheurs de l'Université du Québec à Montréal avaient, eux, saisi 
toute cette logique, dans des textes (Dumas 1983; Morisset 1985) portant sur les Inouites et l'inuktitut (l'inouquetitoute?) du Nunavik (nounavique?).)

Cette querelle des inuitophiles et des francocentristes fait, bien sûr, un peu figure de guerre lilliputienne quand on la compare aux grands débats linguistiques et littéraires d'hier et d'aujourd'hui, la querelle des Anciens et des Modernes du $17^{\mathrm{e}}$ siècle français par exemple. Elle a cependant le mérite de mettre en lumière deux grandes tendances - dont l'une n'éliminera sans doute jamais l'autre - de l'expression écrite contemporaine: l'allégeance première à des critères éthiques, politiquement corrects au bon sens du terme, ou la priorité donnée à des règles arbitraires, peut-être linguistiquement correctes (quoique, comme on l'a vu, ce ne soit pas toujours le cas), mais parfois dérogatoires à l'égard de ceux dont elles permettent de parler. L'équipe de rédaction d'Études/Inuit/Studies - comme de nombreux Inuit et inuitologues francophones - prend clairement parti pour la première de ces tendances.

\section{Références}

BERGERON, Gaston

1994 Télécopie adressée à Louis-Jacques Dorais, Québec, 14 février.

CSONKA, Yvon

1995 Les Ahiarmiut. À l'écart des Inuit Caribous, Neuchâtel, Éditions Victor Attinger.

DORAIS, Louis-Jacques

1974 Qu'est-ce qu'un Inuk? Recherches amérindiennes au Québec, 4(4-5): 77-78.

1975 Qu'est-ce qu'un Inuk? North / Nord, 22(6): 38-41.

1996 La Parole inuit. Langue, culture et société dans l'Arctique nord-américain, Paris, Éditions Peeters.

1998 Les Inouits et les Inouites parlent l'inouquetitoute, Nunatsiaq News, août.

DUMAS, Denis

1983 Deux marques phonologiques de cliticisation en inouit d'Ungava, Revue de l'Association québécoise de linguistique, 3(2): 167-175.

HURET, Pauline (dir.)

2003 Les Inuit de l'Arctique canadien, Québec, CIDEF-AFI.

LAUGRAND, Frédéric

2002 Mourir et renaître. La réception du christianisme par les Inuit de l'Arctique de l'Est canadien (1890-1940), Québec, Les Presses de l'Université Laval. 
MENNECIER, Philippe

1995 Le tunumiisut, dialecte inuit du Groenland oriental. Description et analyse, Paris, Klincksieck.

MORISSET, Jean

1985 L'identité usurpée, Montréal, Nouvelle optique.

NAPPAALUK, Mitiarjuk

2002 Sanaaq, roman translittéré et traduit de l'inuktitut par Bernard Saladin d'Anglure, Montréal, Stanké.

OLF (Office de la langue française)

1993 Termes recommandés et en instance de normalisation, Gazette Officielle du Québec, 125(17): 1793-1795.

ROBBE, Pierre

1994 Les Inuit d'Ammassalik, Chasseurs de l'Arctique, Paris, Éditions du Muséum.

THERRIEN, Michèle

1999 Printemps inuit. Naissance du Nunavut, Montpellier, Indigène Éditions. 